Rev. Adv. Mater. Sci. 57 (2018) 26-34

\title{
THE EFFECT OF CRYSTALLOGRAPHIC ORIENTATION ON THE DEFORMATION MECHANISMS OF NiAI NANOFILMS UNDER TENSION
}

\author{
Karina A. Krylova', Rita I. Babicheva'2, Kun Zhou ${ }^{3}$, Alexey M. Bubenchikov³, \\ Evgenii G. Ekomasov ${ }^{4}$ and Sergey V. Dmitriev ${ }^{1,3}$
}

\author{
${ }^{1}$ Institute for Metals Superplasticity Problems, Russian Academy of Sciences, 39 Khalturin St., \\ Ufa 450001, Russia
}

${ }^{2}$ School of Mechanical and Aerospace Engineering, Nanyang Technological University, 50 Nanyang Avenue, Singapore 639798, Singapore

${ }^{3}$ National Research Tomsk State University, Prosp. Lenina 36, Tomsk 634050, Russia

${ }^{4}$ National Research South Ural State University, 76 Lenin avenue, Chelyabinsk, Russia

Received: February 14, 2018

\begin{abstract}
Molecular dynamics is applied to study the effect of crystallographic orientation on the plastic deformation mechanisms and mechanical properties of $\mathrm{NiAl}$ intermetallic nanofilms subjected to uniaxial tension. It is observed that the deformation mechanisms qualitatively depend on the crystallographic orientation of the nanofilms with respect to the loading direction. Plastic deformation of the nanofilms along [557] crystallographic direction is associated with the edge dislocation sliding in the slip system [001](110). As for the nanofilms stretched along [554] and [111] directions, their deformation occurs first through the dislocation sliding followed by the formation of (112)[11 $\overline{1}$ ] twins. Uniaxial tension of the nanofilms along [559] and [55 11] leads to the nucleation and growth of a martensitic phase followed by their rupture along an interface. The maximum (minimum) strength of 9.9 (7.0) GPa is observed for the nanofilms stretched along the [559] ([554]) crystallographic direction, while the largest (smallest) strain to failure of $27(15) \%$ is for [559] ([55 11]). Various deformation mechanisms of the nanofilms are explained through computing the Schmid factor for the operational slip system. The results indicate that the crystallographic orientation is among the key parameters controlling the deformation mechanisms and mechanical properties of intermetallic nanofilms.
\end{abstract}

\section{INTRODUCTION}

Due to high Young's modulus (240 GPa), melting point (1911K), corrosion resistance and low specific density $\left(5.9 \mathrm{~g} / \mathrm{cm}^{3}\right)$, NiAl intermetallic compounds can find application, for instance, in gasturbine engine manufacturing, heat resistant coatings, and other high-temperature applications [1-3]. Nanomaterials made of $\mathrm{Ni}$-Al alloys possess unique mechanical properties, they can be a part of composite materials [4], their strength can be close to the theoretical value [5-7], these are important fac- tors for further increasing the use of such materials in various applications.

At the same time, low ductility of NiAl materials at room temperature and low strength at increased temperatures [8-10] lead to limitations of their use. Therefore researchers have been looking for approaches to increase strength and ductility of NiAl. For example, ductility up to $139 \%$ for the singlephase Ni-50Al alloy with an average grain size of $220 \mu \mathrm{m}$ was observed during high temperature uniaxial tension at certain strain rate [11]. Such behavior is due to the continuous dynamic recovery 
and recrystallization when low-angle grain boundaries of sub-grains transform to high-angle ones resulting in decrease of the average grain size down to $30 \mu \mathrm{m}$. The superplastic elongation $(\varepsilon>180 \%)$ during tension of the NiAl-9Mo alloy consisting of áMo dispersoid phase and $\beta$-NiAl matrix was shown to be associated with sliding and rotation of matrix grain boundaries [12]. High ultimate compressive strength and strain to failure (2143 MPa and $32.2 \%$, respectively) were demonstrated during deformation of nanocrystalline NiAl subjected to mechanical alloying and high temperature sintering [13]. Intermetallic compounds demonstrate deformation mechanisms different from those of pure metals or disordered alloys [14]. In-situ scanning electron microscopy tensile experiments and molecular dynamics (MD) simulations on nominally defect-free single-crystalline Au nanowhiskers demonstrated that deformation twinning of nanoscale materials can result in their high tensile ductility in addition to ultra-high strength [15]. NiAl single crystals oriented along [213] exhibit tensile elongations of up to $28 \%$ at room temperature under certain conditions [16]. Levit et al. [17] observed more than $300 \%$ elongation of NiAl single crystal during tension along the [557] crystallographic direction. The authors explained this through the contribution to the deformation from the only operational slip system [001](110) which has the maximum Schmid factor of 0.5 . It has been shown experimentally that copper nano-component has a much higher yield stress and hardening rate compared to bulk copper [18]. Grain size effect on plasticity and strength of complex-shaped nanocrystalline nickel pillars was studied in [19].

One of the possible approaches to study the deformation mechanisms in nanomaterials is to use MD. Using this method, for example, it was shown recently that NiAI nanowires having B2 superstructure can demonstrate superplasticity $(\varepsilon \sim 700 \%)$ caused by structure amorphization [20]. Li et al. [21] demonstrated via MD simulations that in bcc nanowires, the superelasticity effect is associated with reversible twinning deformation mechanism. A non-symmetric deformation at tension/compression and pseudoelastic/pseudoplastic behavior of NiAl and $\mathrm{CuZr}$ nanowires due to phase transformations were observed through MD modeling [20-27]. Depending on the strain rate and temperature, different plastic deformation mechanisms of Cu nanowires were revealed [28]. MD simulations of a tensile loading at $300 \mathrm{~K}$ were performed for Fe nanopillars oriented along [001] and [110] directions to demonstrate that in the former case, plastic deformation is initiated by dislocation nucleation at the edges of the nanopillars, whereas in the latter case through a phase transformation inside the nanopillars [29]. With the help of very large scale MD simulations it has been shown that the yield strength of nanomaterials is highly sensitive to their internal and surface structures [30]. Plastic deformation associated with the zigzag stress-strain curves were studied in perfect and surface defected Cu nanowires under their uniaxial tension [31]. It was shown that defects have influence on the nanowire's plastic deformation mechanisms. A drastic temperature softening effect on the film strength and modulus was observed for copper nanofilms deformed at different temperatures and high loading rates [32]. Simulation results reported in [33] showed that not only the yield strength but also the shear modulus of single-crystal copper nanofilms subjected to shear loading increases with decreasing film thickness. MD simulations were used to investigate deformation behavior of $00010 /\{100\}$ single-crystal $\mathrm{Cu}$ nanowires under bending and torsion. For the nanowires subjected to bending, fivefold deformation twins were observed, while for the nanowires under torsion, the plastic deformation mechanism is the creation of full dislocations from the ends of the nanowires [34]. Recently, tensile mechanical behavior of silicon carbide thin films [35] and bi-crystalline Si nanofilms [36] were also investigated by using MD. It was established that the deformation of NiAl and FeAl nanofilms and nanowires during uniaxial tension along the [100] crystallographic direction occurs elastically up to $30-40 \%$ strain through the formation of domains with different strain levels [37-42].

The abovementioned experimental and numerical studies suggest that strength and ductility of $\mathrm{NiAl}$ intermetallic alloys may depend on many parameters, among which crystallographic orientation with respect to the loading direction, which controls the Schmid factor, is one of the key issues $[16,17,43]$. However, to the best of our knowledge, the effect of crystallographic orientation on the deformation mechanisms has not been studied for $\mathrm{NiAl}$ single crystal nanofilms. The current paper addresses this problem with the use of MD simulations.

\section{MODELING}

In this work, nanofilms made of NiAl with B2 superstructure having bcc lattice are chosen as a material of study. The NiAl lattice parameter is $a=2.8712 \AA$. 


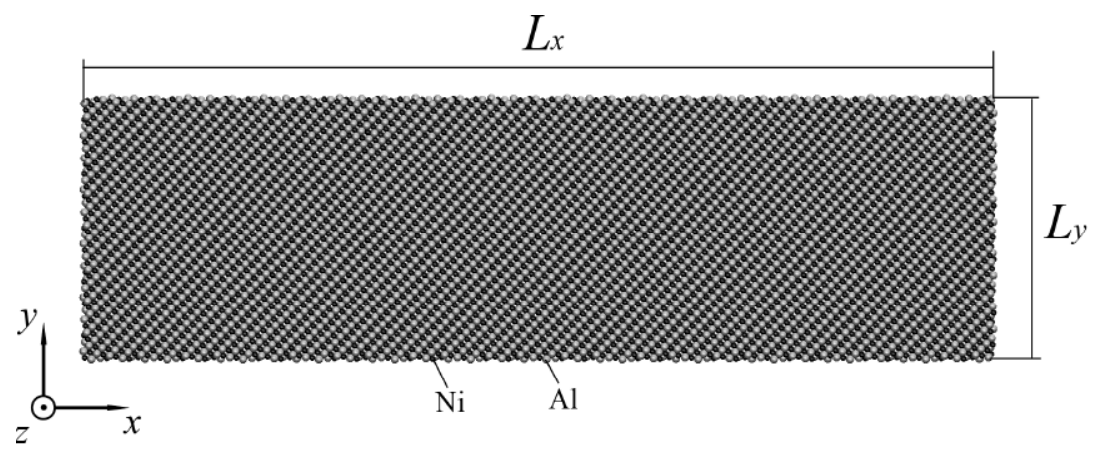

Fig. 1. Example of the computational cell for NiAl nanofilm having the [557] crystallographic direction along the $x$ axis and the $(\overline{1} 10)$ crystallographic plane parallel to the $x y$ plane. Periodic boundary conditions are applied along the $x$ and $z$ directions, while free surfaces are perpendicular to the $y$ axis. Uniaxial tension is applied along $x$ axis. All stress components except for $\sigma_{x x}$ are controlled to be zero.

Fig. 1 shows an example of the computational cell of NiAl nanofilm having the [557] crystallographic direction along loading axis which coincides with the $x$ axis. For all NiAl crystallographic orientations considered, dimensions of computational cells are $L_{x} \approx 340 \AA, L_{y} \approx 95 \AA, L_{z} \approx 17.2 \AA$ along the $x, y$, and $z$ axes, respectively. The use of relatively small size of the computational cell along $z$ direction is justified by the experimental observation that even macroscopic single crystal samples of NiAl with similar crystallographic orientation with respect to the loading direction demonstrate plane strain deformation $[16,17]$. In order to simulate infinite NiAl nanofilms, the periodic boundary conditions are applied along the $x$ and $z$ directions removing such way the surface effect, while free surfaces remain perpendicular to the $y$ axis. For the nanofilm shown in Fig. 1, we have done test simulations for the computational cell with $L_{y} \approx 190 \AA$ and observed no qualitative changes in the plastic deformation because it was controlled by the defect nucleation at the surfaces. We did not consider larger size of the computational cell in the $x$ direction $\left(L_{x}\right)$ because our aim was to study plastic deformation of the nanofilm, while for increasing $L_{x}$ one would expect transition to brittle fracture according to the work [44].

Five different crystallographic orientations of the nanofilms are considered, keeping the [554], [111], [557], [559] or [55 11] direction coinciding with the $x$ axis. For all these orientations, the $(\overline{1} 10)$ crystallographic plane is parallel to the $x y$ plane.

The nanofilms are subjected to uniaxial tension along the $x$ axis. All the stress components except for $\sigma_{x x}$ are controlled to be zero. MD modeling of the nanofilm uniaxial tension is conducted at $10 \mathrm{~K}$ with the strain rate of $\dot{\varepsilon}=10^{8} \mathrm{~s}^{-1}$. Test calculations showed that the decrease of strain rate by one or- der of magnitude does not lead to a noticeable change of results.

MD simulations of the NiAl nanofilm uniaxial tension are performed by using the large-scale atomic/ molecular massively parallel simulator (LAMMPS) program package [45]. The interatomic forces are modeled with the many-body embedded-atom method potentials by Purja Pun and Mishin [46]. These interatomic potentials are chosen because $M D$ results based on the potentials are consistent with experimental studies and ab initio calculations. The Verlet method of order four with the time step of $1 \mathrm{fs}$ is used for numerical integration of the atomic equations of motion.

\section{RESULTS}

Fig. 2 shows stress-strain curves $\sigma_{x x}\left(\varepsilon_{x x}\right)$ obtained during uniaxial tension of the NiAl nanofilms along different crystallographic directions. It is clearly seen that stress-strain relations depend noticeably on the nanofilm orientation and therefore, one can expect that various deformation mechanisms may operate in these cases.

All the nanofilms undergo elastic followed by plastic deformation. For instance, the nanofilm tensioned along the [557] crystallographic direction (thick, solid black line in Fig. 2) elongates elastically up to the strain $\varepsilon_{1}$ and stress $\sigma_{1}$ which corresponds to the nanofilm ultimate strength. Note that due to the nonlinearity of the interatomic potentials, for $\varepsilon_{x x}>0.05$ elastic response of the nanofilm is nonlinear. Analogously, elasticity limit $\varepsilon_{1}$ can be specified for the other nanofilms. The plastic deformation starts with a nucleation of first dislocations at $\sigma_{1}$. Due to initial defect-free structure, the NiAl nanofilms demonstrate very high stresses $\sigma_{1}$ in comparison with conventional bulk materials and this is 


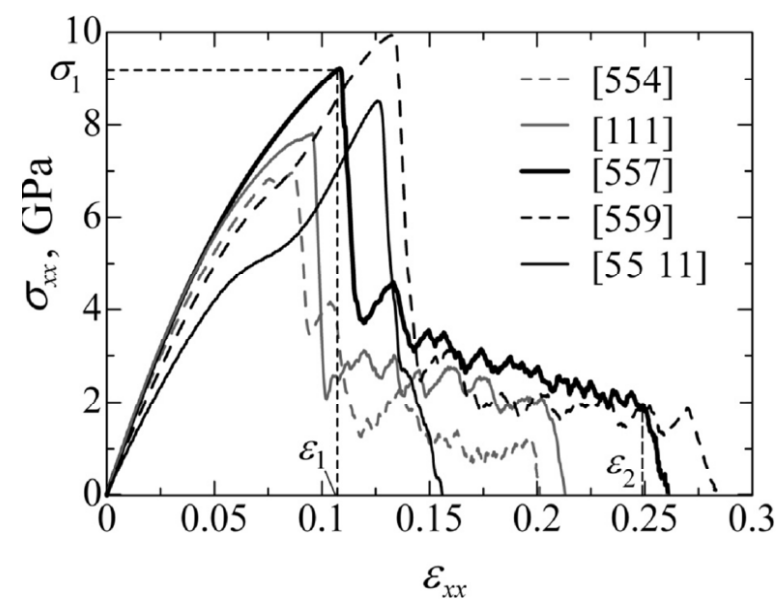

Fig. 2. Stress-strain curves for NiAl nanofilms under uniaxial tension along various crystallographic directions. For the case of [557] loading direction, ultimate stress $\sigma_{1}$, corresponding strain $\varepsilon_{1}$ and strain to failure $\varepsilon_{2}$ are shown.

typical for defect-free metallic nanomaterials [43]. The plastic deformation of the nanofilms occurs up to the strain $\varepsilon_{2}$ (see Fig. 2) followed by their failure. It is clearly seen that the stress-strain relations of the nanofilms depend on their crystallographic orientations with respect to the loading direction. In Table 1, values of $\sigma_{1}, \varepsilon_{1}$, and $\varepsilon_{2}$ for the nanofilms with different orientations are shown. The highest ultimate stress of $\sigma_{1}=9.9 \mathrm{GPa}$ and the strain to failure of $\varepsilon_{2}=0.27$ are observed for the tension along [559] crystallographic direction. The lowest ultimate stress of $\sigma_{1}=7.0 \mathrm{GPa}$ is for the elongation along [554], while the lowest ductility of $\varepsilon_{2}=0.15$ for [55 11].

Structure of nanofilms at different strain levels is analyzed and discussed in Section 4.

\section{DISCUSSION}

It is well-known that the dislocation sliding occurs first in a slip system having the highest Schmid factor, closest to 0.5 . Pure bcc metals have many slip systems, but in ordered alloys with the B2 superstructure some of them do not operate because the dislocation sliding in such systems is accompa-

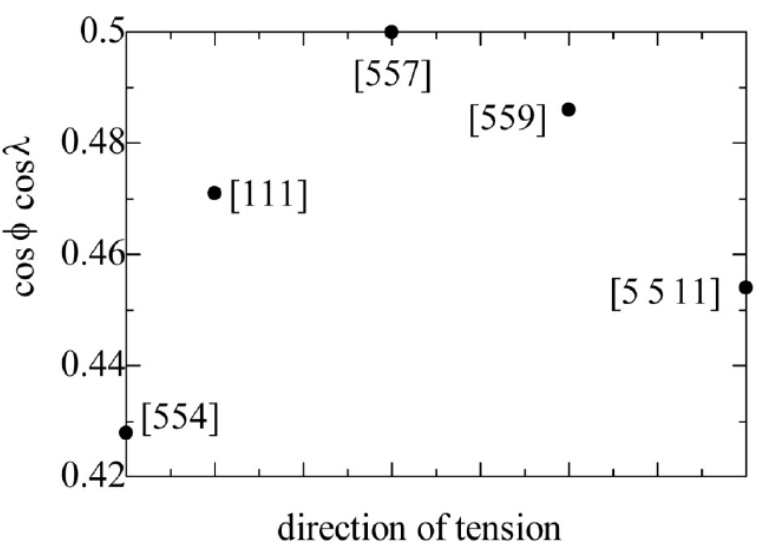

Fig. 3. The Schmid factor for the slip system [001](110) vs loading direction of nanofilms.

nied by formation of antiphase boundaries, i.e., disturbs ordered structure and therefore increases such way the overall energy of the material. According to this criterion, the main slip system in the B2-ordered NiAl is [001](110) [47]. Fig. 3 shows the Schmid factor of this system for different loading directions. The Schmid factor is calculated as $\cos \varphi \cos \lambda$, where $\varphi$ and $\lambda$ are angles between the loading direction considered and the sliding plane (110) and the sliding direction [001], respectively.

Among all the crystallographic orientations, the maximum Schmid factor of 0.5 for the [001](110) slip system is observed upon tension of the nanofilms along the [557] direction, while the minimum value of 0.428 for [554]. From the data shown in Fig. 3, the [001](110) slip system is best activated in the case of tension along the [557] crystallographic direction.

In Fig. 4, the atomic structure of the nanofilm elongated by $\varepsilon_{x x}=0.115$ along the [557] crystallographic direction is shown. Steps at the free surfaces appear as a result of the dislocation sliding in the material. Edge dislocations of the [001](110) slip system are depicted by symbol " $\perp$ ". The absence of the other types of lattice defects indicates that the deformation occurs solely by the dislocation sliding mechanism. The nanofilm under-

Table 1. Ultimate stress $\sigma_{1}$, corresponding strain $\varepsilon_{1}$ and strain to failure $\varepsilon_{2}$ vs loading direction of NiAl nanofilms.

\begin{tabular}{llllll}
\hline & {$[554]$} & {$[111]$} & {$[557]$} & {$[559]$} & {$[5511]$} \\
\hline$\sigma_{1}, \mathrm{GPa}$ & 7.0 & 7.8 & 9.2 & 9.9 & 8.5 \\
$\varepsilon_{1}$ & 0.086 & 0.095 & 0.108 & 0.133 & 0.126 \\
$\varepsilon_{2}$ & 0.19 & 0.20 & 0.25 & 0.27 & 0.15 \\
\hline
\end{tabular}




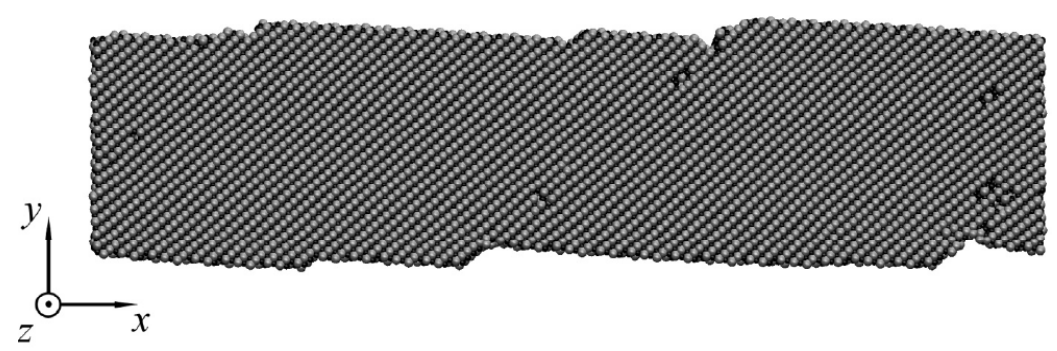

Fig. 4. Computational cell of NiAl nanofilm at $\varepsilon_{x x}=0.115$ applied along the [557] crystallographic direction. Steps on the surface are formed due to nucleation (at the surface) and sliding of dislocations in the [001](110) slip system.

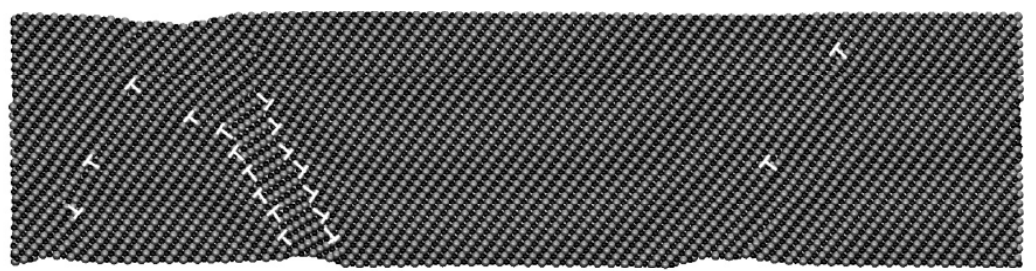

(a) $\varepsilon_{\mathrm{xx}}=0.097$

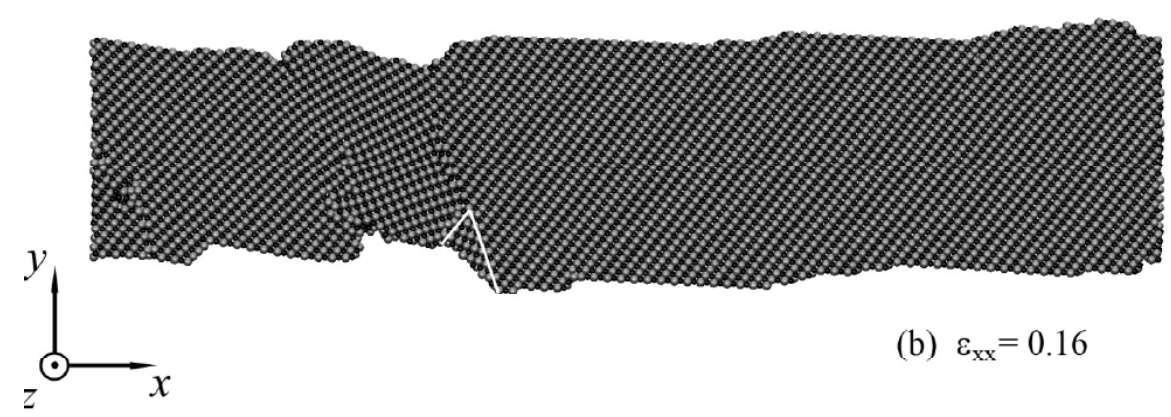

Fig. 5. Computational cells of NiAl nanofilms under tension along [111] at $\varepsilon_{x x}=0.097$ (a) and $\varepsilon_{x x}=0.16$ (b). In (a) edge dislocations sliding in the [001](110) slip system are depicted by symbol “ $\perp$ ". In (b), twin boundaries are shown by white solid lines.

goes failure at $\varepsilon_{x x}=0.25$ along the dislocation sliding direction.

The nanofilms subjected to tension along the [111] and [554] directions are shown in Figs. 5 and 6, respectively. It is seen that in both cases, the deformation occurs first through the dislocation sliding in the [001](110) slip system followed by twinning. Edge dislocations are depicted by symbol “ $\perp$ " (Figs. $5 a$ and 6a). For tension along the [111] and [554] directions, a twin formation can be observed at $\varepsilon_{x x}=0.16$ and $\varepsilon_{x x}=0.11$, respectively (Figs. $5 \mathrm{~b}$ and $6 \mathrm{~b}$, where twin boundaries are indicated by white solid lines). Analysis of a twin structure revealed that the twin plane corresponds to (112) while its shear direction is $[11 \overline{1}]$. Note that new dislocations do not nucleate after the twin formation and further deformation of the nanofilms is only due to a growth of the twins. It is observed that in these cases, failure of the NiAl nanofilms occurs along the twin boundaries.
The deformation process for the nanofilm uniaxial tension along the [559] and [55 11] crystallographic directions is different from the previous two cases. It is seen in Fig. 7 that the loading of the nanofilms along these two directions leads to a transformation of the B2 phase into a new phase (NP). Due to the relatively high Schmid factor for the [559] loading direction (see Fig.3), the dislocation sliding can be seen at early stages of the deformation (Fig. 7a). Note that this mechanism starts to work only after an appearance of the new phase. The dislocations nucleate at the interphase boundaries, unlike all the other cases for which the dislocations form at the free surfaces of the nanofilms. The increase in strain level is accompanied by the growth of a new phase area. For the [55 11] loading direction, the edge dislocations are not seen (Fig. 7b), that can be explained by the low Schmid factor (see Fig. 3). Probably, this is the reason for a low plasticity of the nanofilm when subjected to the extension along this 


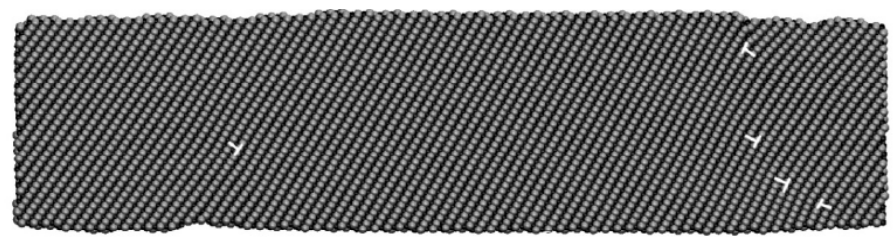

(a) $\varepsilon_{x x}=0.09$

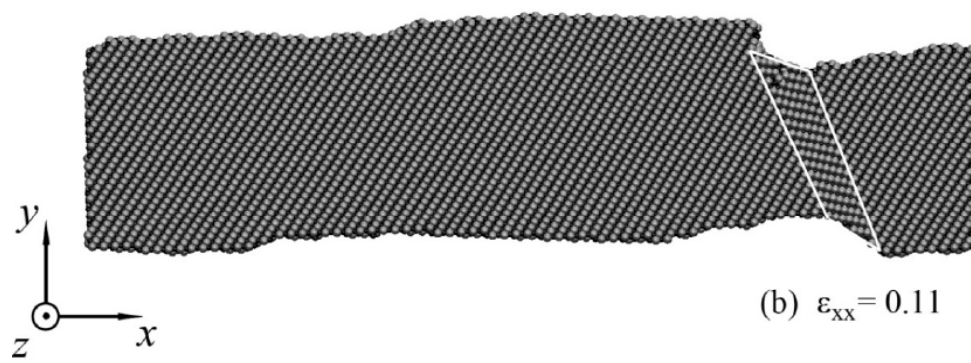

Fig. 6. Computational cells of NiAl nanofilms under tension along [554] at $\varepsilon_{x x}=0.09$ (a) and $\varepsilon_{x x}=0.16$ (b). In (a) edge dislocations sliding in the [001](110) slip system are depicted by symbol “ $\perp$ ". In (b), twin boundaries are shown by white solid lines.

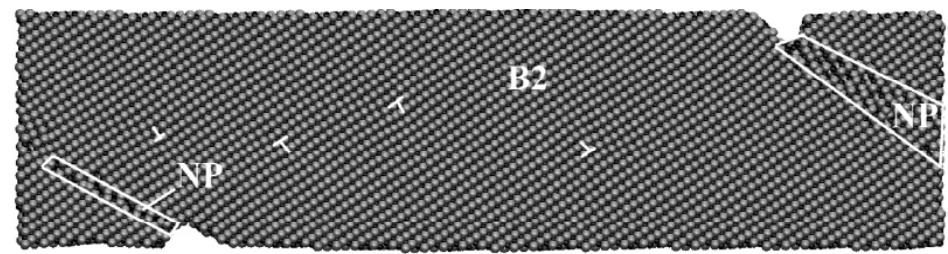

(a) $\varepsilon_{\mathrm{xx}}=0.14$

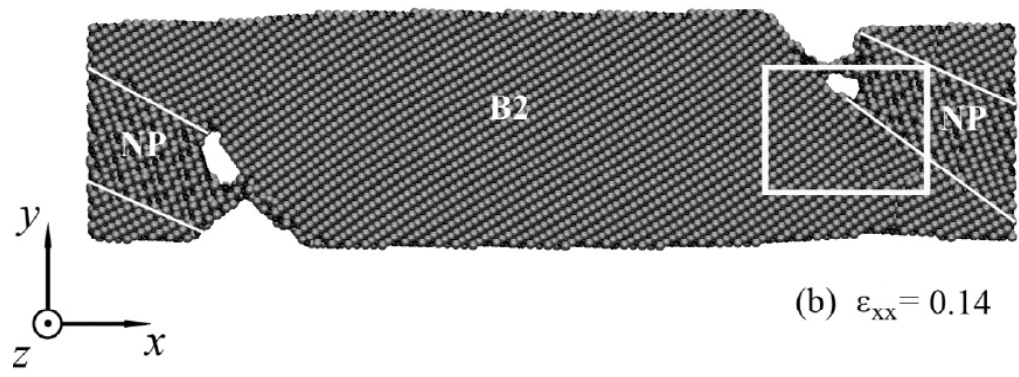

Fig. 7. Computational cells of NiAl nanofilms under tension along [559] (a) and [55 11] (b) at $\varepsilon_{x x}=0.14$. Interphase boundaries between B2 and new phases (NP) are indicated by white solid lines. Nanofilm structure in the framed area in (b) is further analyzed in Fig. 8.

direction (Fig. 2). Fracture of the nanofilms under tension in the [559] and [55 11] directions occurs along the interphase boundaries.

Fig. 8 shows an atomic structure of the area framed in Fig. 7b. Note that the B2 phase and the new phase are on the left bottom and on the right top corners of this area, respectively. The same crystal structure is shown in Figs. $8 \mathrm{a}$ and $8 \mathrm{~b}$ after a rigid rotation to reveal the atomic planes. The primitive translational cell for the B2 phase in the form of a cube is highlighted by the black square in Fig. $8 \mathrm{a}$, while that for the new phase is shown by a parallelogram in Fig. 8b. The structure analysis of the new phase revealed that it is the stress-induced martensite. The original tetragonal unit cell of the
B2 structure (Fig. 9a) transforms to the martensite lattice having an angle $\alpha \approx 34^{\circ}$ between the initial $c$ axis and the $c^{\prime}$ axis as shown in Fig. 9b. Similar phase transformation was observed in NiTi shape memory alloys [48-53]. A formation of the martensite phase in NiAl alloys was studied experimentally by Chakravorty et al. $[54,55]$ and via MD simulations of the NiAl nanowire tension [56]. The modeling in the latter case was performed by using the many-body potential by Voter and Chen $[57,58]$.

\section{CONCLUSIONS}

The deformation mechanisms and mechanical properties of the B2-ordered NiAl single crystal nanofilms 

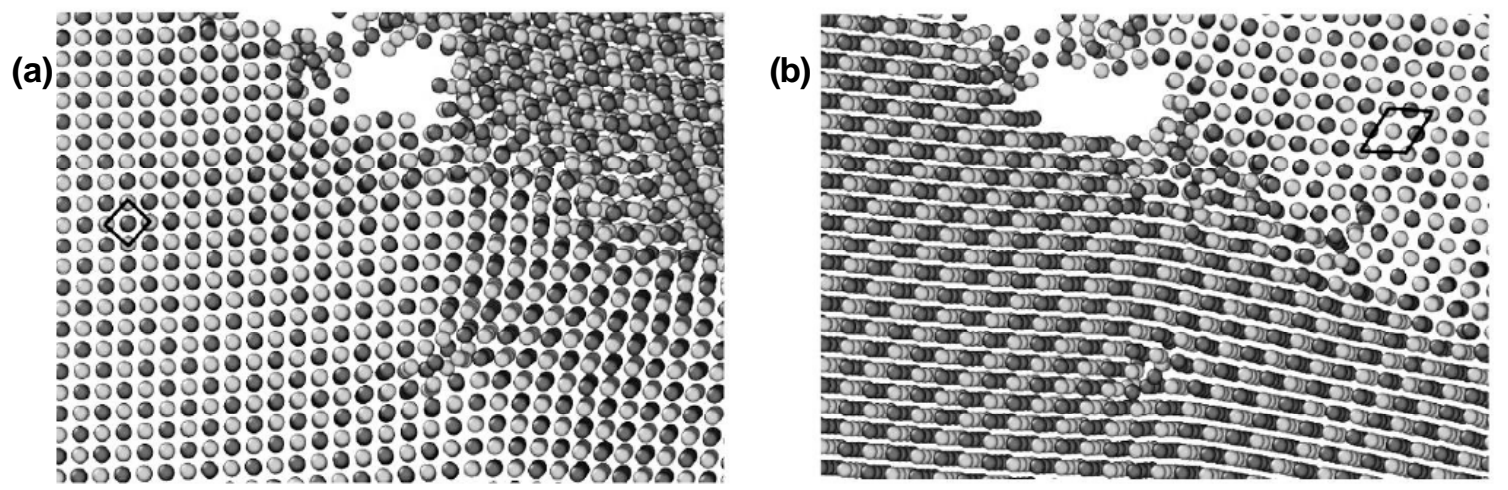

Fig. 8. Enlarged framed area in Fig. 7b. The same structure is shown in (a) and (b) after a rigid rotation to reveal the atomic planes. Primitive translational cell for the B2 phase is shown in (a) and that of the new phase in (b). The cell in (a) contains two atoms, while in (b) four atoms.
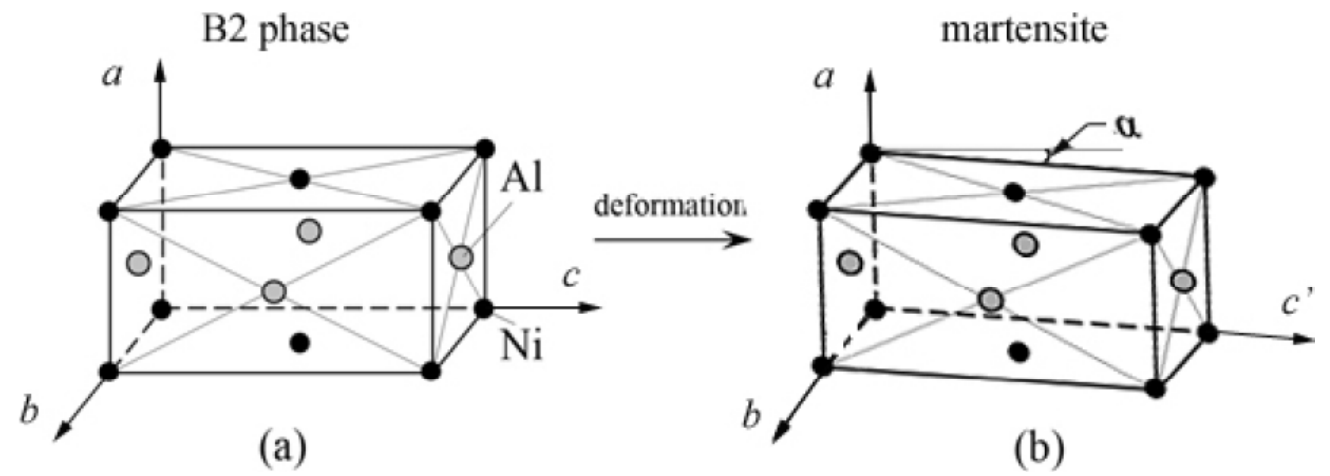

Fig. 9. (a) Original tetragonal unit cell of the cubic B2 structure (replotted data from [48]) and (b) the structure of the martensite phase formed during tension of NiAl nanofilms along the [559] and [55 11] crystallographic directions.

subjected to the uniaxial tension when the loading direction lies in the (10) plane and coincides with the [554], [111], [557], [559] or [55 11] crystallographic direction are studied. The only operational slip system in all the cases is [001](110) and the highest Schmid factor of 0.5 is realized for the tension along [557] (Fig. 3). The deformation mechanisms observed in the differently oriented nanofilms are found to be strikingly different.

As expected, for the tension along the [557] axis with the highest Schmid factor, the dislocation sliding plays the dominant role (Fig. 4). A deviation of the tensile direction toward smaller values of the third Miller index ([554] and [555] = [111]) results in a reduction of the contribution from the dislocation sliding, especially for the tension along [554] with the lowest Schmid factor among the considered cases. The dislocation sliding observed for a relatively small strain $\left(\varepsilon_{x x}<0.1\right)$, switches to the deformation via the (112)[11 $]$ ] twinning at higher strain (see Figs. 5 and 6). Similarly, the contribution from the dislocation sliding decreases with the deviation of the tensile direction from [557] toward larger val- ues of the third Miller index ([559] and [55 11]). The difference is that not the twinning but the martensite transformation takes place at relatively high elongation strains (Fig. 7).

The crystallographic orientation dependence of the mechanical properties of the nanofilms can be related to the operating deformation mechanisms. The films extended along the [557] and [559] directions exhibit the highest strength and ductility (Fig. 2 and Table 1). Recall that for these samples the Schmid factor is higher than for the other nanofilms (Fig. 3). The ease of the dislocation sliding in the nanofilms extended along the [557] and [559] directions is the reason for a more homogeneous plastic deformation in comparison with the other cases. Suppression of the dislocation sliding results in the appearance of either twins or martensite phase, which makes the deformation highly nonhomogeneous and results in a relatively early failure of the nanofilms along the interface boundaries.

The results presented here differ significantly from those obtained in our previous studies [38-43] where 
tension of NiAl nanofilms along the [100] direction was considered and a nonhomogeneous elastic strain up to 0.35 was revealed.

Note that the intermetallic compounds possess fewer number of operational slip systems in comparison with pure metals, and this is the explanation why the effect of crystallographic orientation of intermetallic nanofilms and nanowires on their plasticity should be more pronounced than for pure metals or disordered alloys.

Summing up, the results of the current study demonstrate that crystallographic orientation is a very important parameter controlling the plastic deformation mechanisms and mechanical properties, especially for intermetallic nanofilms and nanowires. Optimizing the crystallographic orientation of intermetallic nanomaterials with respect to a loading direction one can further improve their strength and ductility.

\section{ACKNOWLEDGEMENTS}

B.R.I. thanks the Russian Science Foundation [grant No. 17-79-10410] for the financial support. S.V.D. appreciates the financial support provided by the Russian Science Foundation [grant No. 14-1300982]. E.G.E. thanks Act 211 Government of the Russian Federation [contract ${ }^{1}$ 02.A03.21.0011] for the financial support.

\section{REFERENCES}

[1] N.S. Stoloff, C.T. Liu and S.C. Deevi // Intermetallics 8 (2000) 1313.

[2] J. Guo, K. Huai, X. Du and R.Yang // Mater. Design 28 (2007) 1940.

[3] R.C. Reed, The superalloys fundamentals and applications )Cambridge University Press; Cambridge, UK, 2006).

[4] N. Thiyaneshwaran, K. Sivaprasad and B. Ravisankar // Int. J. Adv. Manuf. Technol. 93 (2017) 361.

[5] T. Zhu and J. Li // Prog. Mater. Sci. 55 (2010) 710.

[6] A.M. Iskandarov, S.V. Dmitriev and Y. Umeno // Phys. Rev. B 84 (2011) 224118.

[7] K.A. Bukreeva, A.M. Iskandarov, S.V. Dmitriev, Y. Umeno and R.R. Mylyukov // Phys. Solid State 56 (2014) 423.

[8] A. Lasalmonie // Intermetallics 14 (2006) 1123.

[9] D. Miracle // Acta Metall. Mater. 41 (1993) 649.

[10] D. Geist, C. Gammer, C. Rentenberger and H.P. Karnthaler // J. Alloy Compd. 621 (2015) 371.
[11] J. Hu and D. Lin // J. Alloy Compd. 426 (2006) 162.

[12] X.H. Du, J.T. Guo and B.D. Zhou // Mater. Lett. 52 (2002) 442.

[13] E. Liu, J. Jia, Y. Bai, W. Wang and Y. Gao // Mater. Design 53 (2014) 596.

[14] E. Klay, F. Diologent, A. Durussel and A. Mortensen // Intermetallics 19 (2011) 988.

[15] A. Sedlmayr, E. Bitzek, D.S. Gianola, G. Richter, R. Mönig and O. Kraft // Acta Mater. 60 (2012) 3985.

[16] V.I. Levit, I.A. Bul, J. Hu and M.J. Kaufman // Scripta Mater. 34 (1996) 1925.

[17] V.I. Levit, J.S. Winton, Yu. N. Gornostyrev and M.J. Kaufman, In: Proc. Of ReX'96, The Third International Conference on Recrystallization and Related Phenomena (1997), p. 637.

[18] Y. Takahashi, H. Hirakata and T. Kitamura // Thin Solid Films 516 (2008) 1925.

[19] B.B. Seo, J. Gu, Z. Jahed and T.Y. Tsu // Thin Solid Films 621 (2017) 178.

[20] V.K. Sutrakar and R.D. Mahapatra // Mater. Lett. 64 (2010) 879.

[21] S. Li, X. Ding, J. Deng, T. Lookman, J. Li, X. Ren, J. Sun and A. Saxena // Phys. Rev. B82 (2010) 205435.

[22] V.K. Sutrakar and D.R. Mahapatra // Intermetallics 1 (2010) 1565.

[23] V.K. Sutrakar and D.R. Mahapatra // Nanotechnology 20 (2009) 295705.

[24] V.K. Sutrakar and D.R. Mahapatra // Mater. Lett. 63 (2009) 1289.

[25] V.K. Sutrakar and D.R. Mahapatra // J. Phys. Condens. Mat. 20 (2008) 335206.

[26] H.S. Park, K. Gall and J.A. Zimmerman // Phys. Rev. Lett. 95 (2005) 255504.

[27] W. Liang, M. Zhou and F. Ke // Nano Lett. 5 (2005) 2039.

[28] H. Xie, F. Yin, T. Yu, G. Lu and Y. Zhang // Acta Mater. 85 (2015) 191.

[29] Y. Zhang, D.J. Yu and K.M. Wang // J. Mater. Sci. Technol. 28 (2012) 164.

[30] Z.X. Wu, Y.W. Zhang, M.H. Jhon, J.R. Greer and D.J. Srolovitz // Acta Mater. 61 (2013) 1831.

[31] H.F. Zhan, Y.T. Gu, C. Yan, X.Q. Feng and P.K.D.V. Yarlagadda // Comp. Mater. Sci. 50 (2011) 3425.

[32] Y. Gan and J.K. Chen // Mech. Res. Commun. 36 (2009) 838.

[33] X.R. Zhuo and H.G. Beom // Comp. Mater. Sci. 99 (2015) 390. 
[34] X. Tian, J. Cui, C. Zhang, Z. Ma, R. Wan and Q. Zhang // Comp. Mater. Sci. 83 (2014) 250.

[35] W.X. Wang, L.S. Niu, Y.Y. Zhang and E.Q. Lin // Comp. Mater. Sci. 62 (2012) 195.

[36] P. Lin, R.I. Babicheva, M. Xue, H.S. Zhang, H. Xu, B. Liu and K. Zhou // Comp. Mater. Sci. 96 (2015) 295.

[37] R.I. Babicheva, K.A. Bukreeva, S.V. Dmitriev, K. Zhou and R.R. Mulyukov // Computational Methods in Science and Technology 19 (2013) 127.

[38] K.A. Bukreeva, R.I. Babicheva, S.V. Dmitriev, K. Zhou and R.R. Mulyukov // Phys. Solid State 55 (2013) 1963.

[39] K.A. Bukreeva, R.I. Babicheva, S.V. Dmitriev, K. Zhou and R.R. Mulyukov // JETP Lett. 2 (2013) 91.

[40] K.A. Bukreeva, R.I. Babicheva, A.B. Sultanguzhina, S.V. Dmitriev, K. Zhou and R.R. Mulyukov // Phys. Solid State 56 (2014) 1157.

[41] R.I. Babicheva, M.D. Starostenkov and K. Zhou // Letters on Materials 4 (2014) 302.

[42] R.I. Babicheva, K.A. Bukreeva, S.V. Dmitriev, R.R. Mulyukov and K. Zhou // Intermetallics 43 (2013) 171.

[43] C. R. Weinberger and W. Cai // J. Mater. Chem. 22 (2012) 3277.

[44] Z. Wu, Y.W. Zhang, M.H. Jhon, H. Gao and D.J. Srolovitz // Nano Lett. 12 (2012) 910.
[45] S. Plimpton // J. Comput. Phys. 117 (1995) 1.

[46] G.P. Purja Pun and Y. Mishin // Phil. Mag. 89 (2009) 3245.

[47] M.G. Mendiratta and C.C. Law // J. Mater. Sci. 22 (1987) 607.

[48] R.I. Babicheva and Kh.Ya. Mulyukov // Appl. Phys. A Mater. 116 (2014) 1857.

[49] R.I. Babicheva, I.Z. Sharipov and Kh.Ya. Mulyukov // Phys. Solid State 53 (2011) 1947.

[50] R.I. Babicheva, Kh.Ya. Mulyukov, I.Z. Sharipov and I.M. Safarov // Phys. Solid State 54 (2012) 1480.

[51] Kh.Ya. Mulyukov and R.I. Babicheva // Letters on Materials 2 (2012) 186, In Russian.

[52] R.I. Babicheva and Kh.Ya. Mulyukov // Letters on Materials 1 (2011) 55, In Russian.

[53] R.I. Babicheva and Kh.Ya. Mulyukov // Letters on Materials 1 (2011) 156, In Russian.

[54] S. Chakravorty and C.M. Wayman // Metall. Trans. A 7 (1976) 555.

[55] S. Chakravorty and C.M. Wayman // Metall. Trans. A 7 (1976) 569.

[56] H.S. Park // Nano Lett. 6 (2006) 958.

[57] S.P. Chen, A.F. Voter and D.J. Srolovitz // Phys. Rev. Lett. 57 (1986) 1308.

[58] A.F. Voter and S.P. Chen // Mater. Res. Soc. Symp. Proc. 82 (1987) 175. 